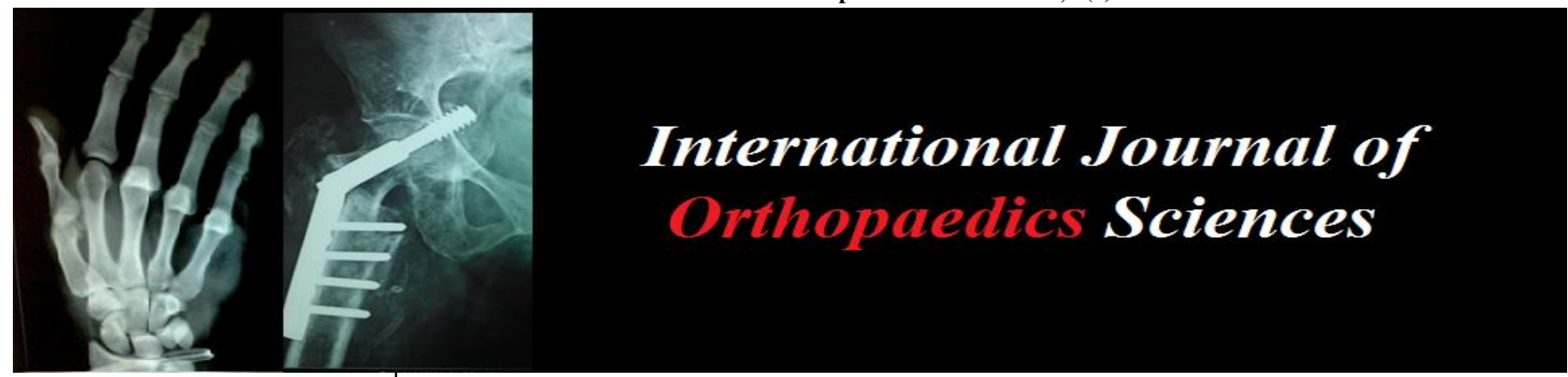

ISSN: 2395-1958

IJOS 2019; 5(3): 551-553

(C) 2019 IJOS

www.orthopaper.com

Received: 06-05-2019

Accepted: 10-06-2019

Dr. Jampani Ravitheja Orthopaedic Surgeon, Manipal Hospital Vijayawada, Andhra Pradesh, India

Dr. Nallamothu Jagadeesh Chief Orthopaedic, Joint Replacment Surgeon, Manipal hospital, Vijayawada, Andhra Pradesh, India

Dr. Nayanatara

Department of General Medicine,

Kasturba Medical College,

Manipal, Karnataka, India

Dr. Jayakrishnan KNK Department of Orthopaedics, Kasturba Medical College, Manipal, Karnataka, India

Correspondence

Dr. Jampani Ravitheja

Orthopaedic Surgeon, Manipal

Hospital Vijayawada, Andhra

Pradesh, India

\section{Fairy tale knee arthroplasty}

\section{Dr. Jampani Ravitheja, Dr. Nallamothu Jagadeesh, Dr. Nayanatara and Dr. Jayakrishnan KNK}

DOI: https://doi.org/10.22271/ortho.2019.v5.i3j.1588

\section{Abstract}

Introduction: Knee arthroplasty is one of the most common procedures done in modern day orthopaedic practice. Q angle is net vector of forces acting over patella for a smooth knee range of movement. Knee arthroplasty in case of deformities should focus of alignment in particular.

Case presentation: A 65 year old male came with operated knee with difficulty in flexion and extension of knee. He was evaluated and found to have femoral component loosening and patellar maltracking and multiple comorbidities. We had a comprehensive preoperative plan and executed it. We treated comorbidities and stabilised the patient. Later we went ahead with revision of femoral component and correction of Q angle. Post-operative patient activities of daily living improved. We followed him for 6 months.

Conclusion: Evaluation and achieving proper alignment of limb in case of deformed knee while performing knee arthroplasty is prime focus. Q angle and its correction will help in restoration of kinematics if there is deformed knee preoperatively.

Keywords: Knee arthroplasty in deformities, Q angle in knee arthroplasty patellar maltracking

\section{Introduction}

Orthopaedic practice and geriatric practice goes hand in hand. Most of orthopaedic surgical procedures are done in geriatric population due various reasons of Fragility and Degenerative disorders ${ }^{[1]}$.

Knee arthroplasty is one of the most common procedures done in modern day orthopaedic practice. Prime focus of knee arthroplasty is on alignment of knee that balances knee kinematics. Knee arthroplasty in a deformed femur and tibia is a challenging task. We would report a case of deformed femur and tibia which had undergone knee arthroplasty and presented to us with severe pain and instability.

\section{Case report}

$65 y$ r old male underwent primary knee arthroplasty 6 months back elsewhere presented to us with postop pain and instability in knee with limited range of movement. He was evaluated with thorough clinical examination and previous radiographs.

History - patient had a history of fracture around knee joint which involved fracture of both femur and tibia frasiers type $1^{[2]}$. He was managed conservatively for 8 months and then and patient continued to walk with stiff knee for almost 20 years \{Fig 1, Fig 2, Fig 3 \}.

Six months prior to presenting to us patient underwent primary knee arthroplasty ${ }^{[4]}$ with LCS elsewhere for stiff knee and pain. Postoperatively he was able to walk but had difficulty in walking and using restroom and had severe pain and difficulty in knee while sitting in chair and in restroom. We evaluated him clinically and radiologically.

\section{Findings}

1. Loosening of femoral component.

2. Patella maltracking

3. Apprehension test positive for patella

4. Alcoholic liver disase 
5. Uncontrolled diabetes \{Glucated haemoglobin is 12 \}

6. Chronic kidney disease with anemia \{Haemoglobin 8.5

Refer fig 4, 5, 6

$\mathrm{Q}$ angle ${ }^{[3]}$ at presentation was 22. Range of movement of knee 0- 40 degree.

\section{Case discussion}

\section{Preoperative plan}

- Diabetic control ${ }^{[7,8]}$

- Monitor hepatic status ${ }^{[9]}$

- Correction of Q-angle with tibial tubercle osteotomy and medialisation ${ }^{[5]}$.

- Revision femoral component and cementation ${ }^{[6]}$

- Safe and fast surgery in view of multiple comorbidities [10]

- Execution of procedure

- Preoperative diabetic control was attained with human actrapid infusions ${ }^{[7]}$

- Safe antibiotic and pain medication usage

- Reopen knee with medial parapatellar approach and revision of femoral component with cementation.

- Careful tibial tubercle osteotomy and medialisation with cc screws [fig 9, 10, 11]
- Intraoperative range of movement assessment

- Total duration of procedure $2 \mathrm{hrs} 15 \mathrm{mins}$.

Knee was mobilised from day 1 post op. postoperative Q angle is 11 degree. For first 3 weeks only $0-30$ rom is planned and later 0-90 is planned. Post-operative patient attained 0-90 rom 5 weeks after surgery and painless knee. We periodically managed patient diabetes and after 12 weeks his glycated haemoglobin was $7.5^{[7,8]}$.

\section{Clinical message/conclusion}

Thorough evaluation of patient before surgery in case of deformities is a must. Q angle is always important in knee arthroplasty. Due to old fracture which was treated conservatively and looking at deformity it can be pointed that soft tissues around knee are mostly fibrosed thus and LCS implant is used for primary surgery. Since there is gross deformity in distal femur and proximal tibia there is always a chance of patella maltracking. Armamentarium of instruments at door step. Proper preop and post op counseling and monitoring inview of multiple comorbidities with interdepartmental cooperation.
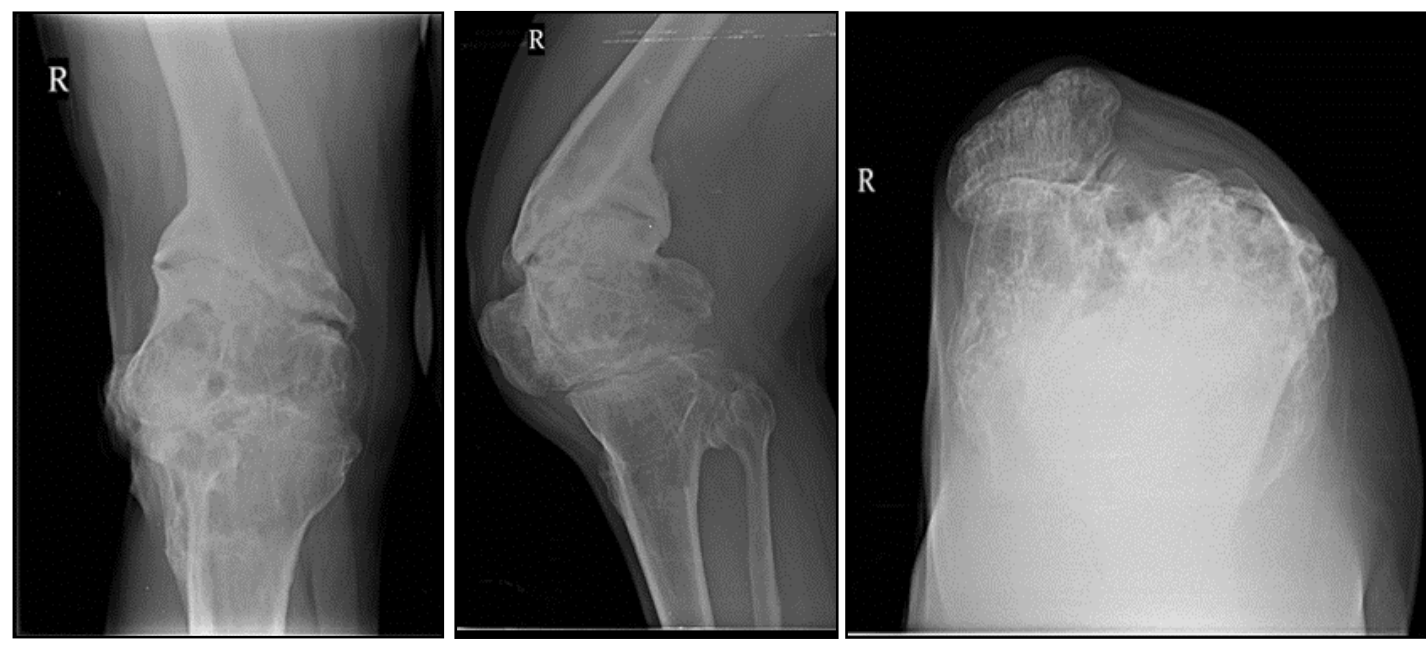

Fig 1, 2, 3: Knee xrays before primary knee arthroplasty
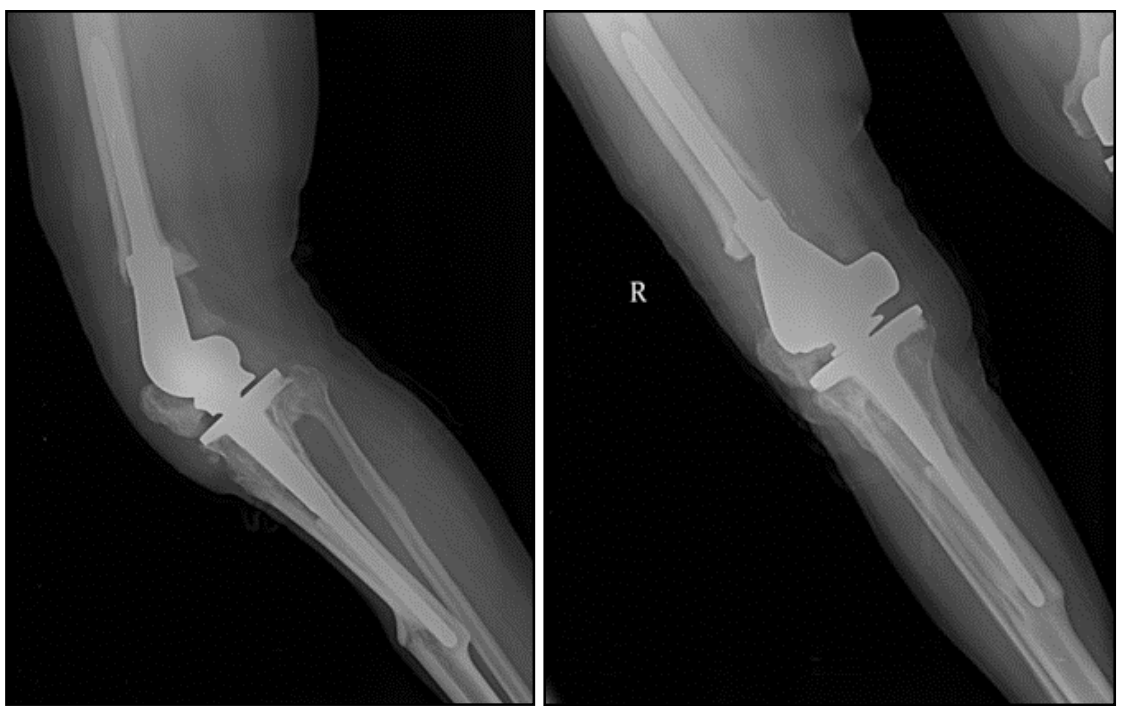

Fig 4, 5: Knee radiographs after primary knee arthroplasty 

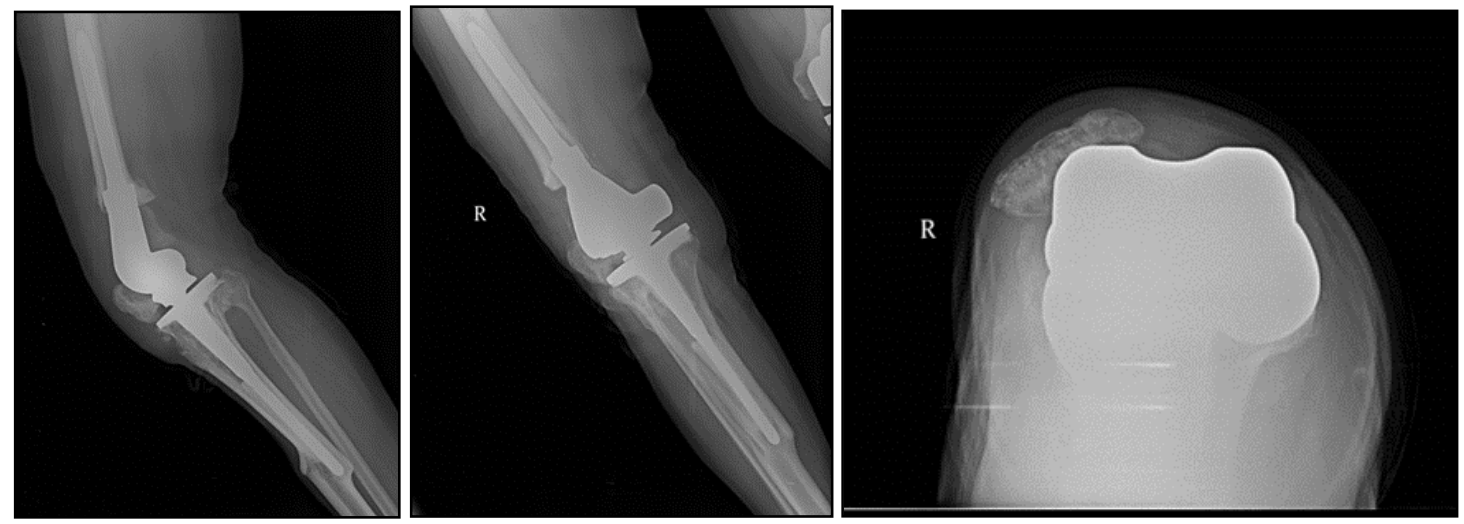

Fig 6, 7, 8: Knee radiographs when presented to us
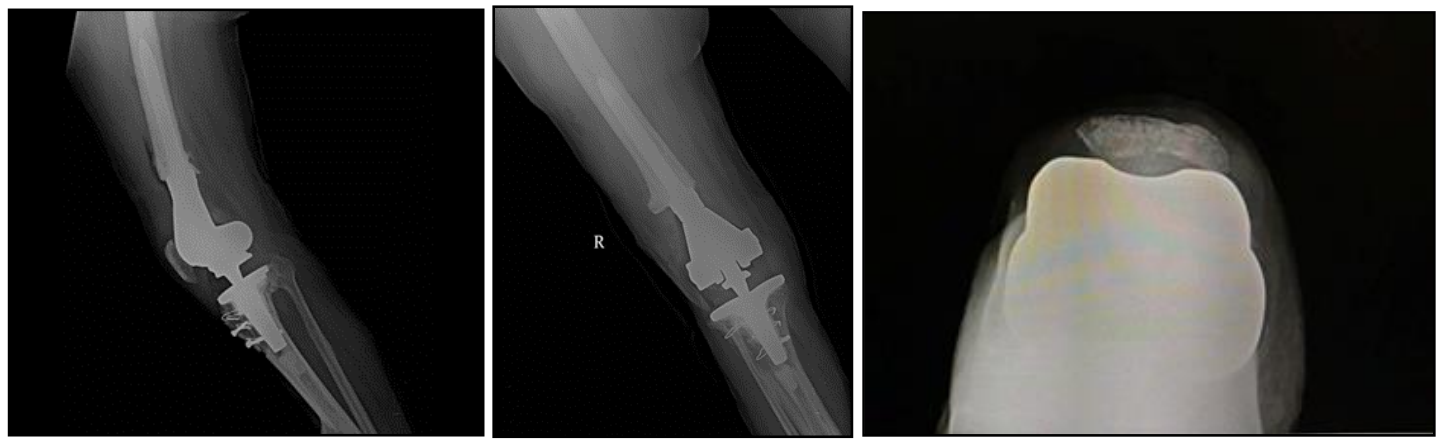

Fig 9, 10, 11: Final radiographs after revision surgery with clinical pics
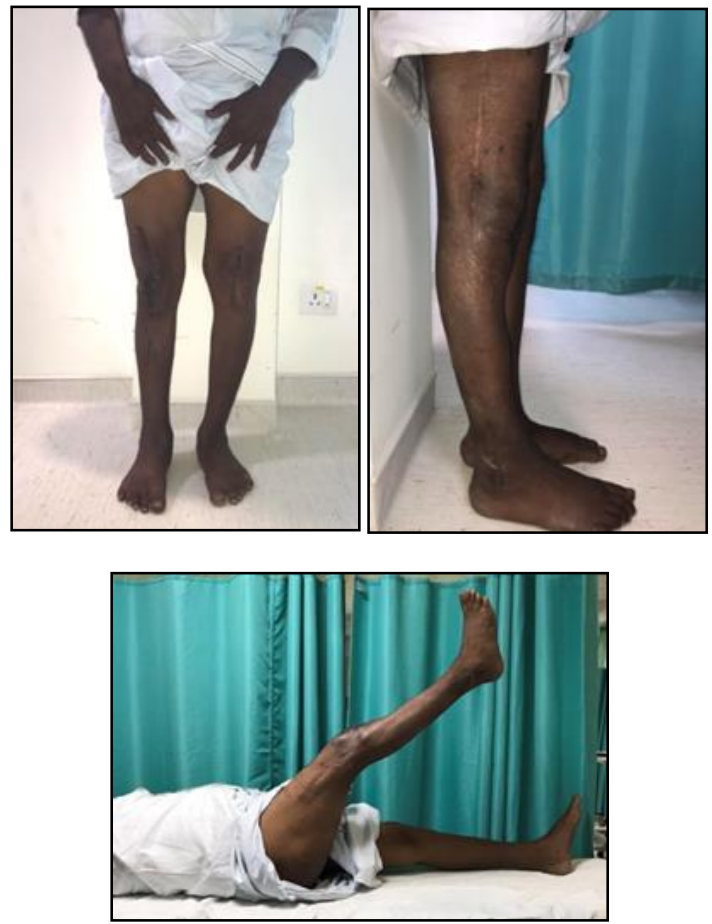

Clinical follow up pictures

\section{References}

1. Quatman C, Switzer J. Geriatric Orthopaedics: a new paradigm for management of older patients. Current geriatrics reports. 2017; 6(1):15-19.

2. Ran T, Hua X, Zhenyu Z, yue L, youhua W, Yi C et al. Floating knee: A modified fraser's classification and the results of a series of 28 cases. Injury. 2013; 44(8):10331042.3.

3. Weiss L, DeForest B, Hammond K, Schilling B, Ferreira L. Reliability of goniometry-based q-angle. PM\&R. 2013; 5(9):763-768.
4. Tsagozis P, Gkavardina A. The Use of Megaprostheses for Reconstruction of Large Skeletal Defects in the Extremities: A critical review. The open orthopaedics journal. 2014; 8(1):384-389.

5. Ferrari M, Sanchez G, Kennedy N, Sanchez A, Schantz $\mathrm{K}$, Provencher M. Osteotomy of the tibial tubercle for Anteromedialization. Arthroscopy techniques. 2017; 6(4):e1341-e1346.

6. Matassi F, Carulli C, Civinini R, Innocenti M. Cemented versus cementless fixation in total knee arthroplasty. Joints. 2014; 1(3):121-5

7. Elgzyri T. Basic management of diabetes mellitus: Practical guidelines. Libyan journal of medicine. 2006; 1(2):176-184.

8. Shah S, Benroubi M, Borzi V, Gumprecht J, Kawamori $\mathrm{R}$, Shaban J et al. Safety and effectiveness of biphasic insulin aspart 30/70 (NovoMix®30) when switching from human premix insulin in patients with type 2 diabetes: subgroup analysis from the 6-month IMPROVETM observational study. International journal of clinical practice. 2009; 63(4):574-582.

9. Andrade R, Tulkens P. Hepatic safety of antibiotics used in primary care. Journal of antimicrobial chemotherapy. 2011; 66(7):1431-1446.

10. Cheng H, Chen B, Soleas I, Ferko N, Cameron C, Hinoul P. Prolonged operative duration increases risk of surgical site infections: A systematic review. Surgical infections. 2017; 18(6):722-735. 